\title{
Medidas de conservación ex situ: Un enfoque metapoblacional a partir del modelo clásico de Levins
}

\section{Ex situ conservation measures: A metapopulation approach based on the classic Levins model}

\author{
Héctor Rojas-Castro ${ }^{1 *}$ \& Sandra Araya-Crisóstomo ${ }^{1}$ \\ ${ }^{1}$ Facultad de Ciencias Básicas, Universidad Católica del Maule, Avenida San Miguel 3605, Casilla 617, Talca, Chile. \\ *horjas@ucm.cl
}

\begin{abstract}
RESUMEN
Dada una metapoblación, que como tal, se encuentra distribuida en fragmentos o parches, podemos pensar que esta está en riesgo de extinción si la cantidad de parches ocupados está disminuyendo. En este sentido, una medida de conservación a considerar podría ser la incorporación de individuos a parches desocupados. Este proceso es conocido como conservación ex situ. En el presente trabajo supondremos una metapoblación extinguiéndose, cuya dinámica será modelada matemáticamente a través del modelo planteado por Richard Levins en su trabajo "Some demographic and genetic consequences of environmental heterogeneity for biological control". Se incorporará al modelo el efecto de una medida de conservación ex situ, consistente en la colonización artificial de parches desocupados, con el propósito de evitar que la población total se extinga. La incorporación de esta medida de conservación será expresada a través de un sistema de ecuaciones diferenciales impulsivo, a partir del cual se generarán una serie de simulaciones, en orden a entender la dinámica a largo plazo y determinar qué factores influyen en el éxito de la medida de conservación.
\end{abstract}

Palabras claves: conservación, ecuaciones diferenciales impulsivas, metapoblación, modelo de Levins.

\begin{abstract}
Given a metapopulation, which as such, is distributed in fragments or patches, we can think that this is at risk of extinction if the number of occupied patches is decreasing. In this sense, a measure of conservation to consider could be the incorporation of individuals to unoccupied patches. This process is known as ex situ conservation. In the present work we will suppose a metapopulation that is becoming extinct, whose dynamics will be modeled mathematically through the model proposed by Richard Levins in his work "Some demographic and genetic consequences of environmental heterogeneity for biological control". The effect of an ex situ conservation measure, consisting of the artificial colonization of unoccupied patches, will be incorporated into the model, with the purpose of preventing the total population from becoming extinct. The incorporation of this conservation measure will be expressed through a system of impulsive differential equations, from which a series of simulations will be generated, in order to understand the long-term dynamics and determine what factors influence the success of the measure of conservation.
\end{abstract}

KEYwords: conservation, impulsive differential equations, Levins model, metapopulation.

\section{INTRODUCCIÓN}

\section{CONCEPTO DE METAPOBLACIÓN}

La Biología de Metapoblaciones estudia los efectos de la dinámica local de poblaciones sobre la persistencia regional de una especie y, por lo tanto, ha alcanzado su máxima aplicación en la Biología de la conservación (Hanski \& Simberloff 1977).

La teoría de metapoblaciones tiene su origen en los trabajos de Sewall Wright, quien demostró teóricamente que la dinámica de las frecuencias génicas puede ser muy diferente en una metapoblación que en una población homogéneamente distribuida en el espacio (Rodríguez 2006). Con posterioridad, Richard Levins elaboró un modelo que demuestra que especies destinadas a la extinción cuando se encuentran homogéneamente distribuidas, pueden sobrevivir regionalmente cuando forman metapoblaciones (Levins 1969). 
La posición espacial de los individuos y poblaciones suelen formar redes de poblaciones conectadas por dispersión, las que se visualizan como conexiones de parches de hábitats de poblaciones locales. De esta manera, la distribución de las poblaciones influye en la tasa de crecimiento y en sus interacciones como: competencia, depredador-presa y huésped-parásito, entre otras.

Ahora bien, si una población es una colección de individuos, una metapoblación es un conjunto de poblaciones locales dentro de un área más extensa, que están conectadas a través de una migración limitada (Boyle 2003).

La definición de Mccullough resulta ser una buena aproximación, ya que además pone énfasis en aspectos biológicos importantes. Al respecto señala que "una metapoblación es una población local que presenta una distribución discontinua. Esta se distribuye sobre fragmentos disyuntivos de hábitat adecuado separados por parches de hábitats inadecuados dentro de los cuales los animales no pueden establecerse. Debido al riesgo de mortalidad que representa el cruzar las condiciones hostiles de la matriz, el movimiento de los animales entre los parches no es rutinario; consecuentemente el movimiento entre fragmentos (dispersión) es restringido" (Mccullough 1996).

\section{CARACTERÍSTICAS DE UNA METAPOBLACIÓN}

Existen importantes diferencias entre la dinámica de un conjunto de poblaciones locales y la de una población única y continua. En primer lugar, en el enfoque metapoblacional, son las poblaciones locales las unidades de observación, y no los individuos, lo que significa que procesos como extinciones y colonizaciones, tienen un mayor impacto en la dinámica poblacional, en relación con los parámetros demográficos como natalidad y mortalidad. Es muy probable que muchos fragmentos adecuados sean pequeños y soporten pequeñas poblaciones, por lo que la extinción en estos parches (extinción local) puede ser un evento común. Por lo tanto, la persistencia de la metapoblación depende de la dinámica de extinción y recolonización entre los diferentes fragmentos a través de la dispersión.

Los modelos de dinámica poblacional exponencial y logístico suponen que las poblaciones son cerradas, sin inmigración o emigración y que, por lo tanto, el crecimiento poblacional varía únicamente en función de los procesos de natalidad y mortalidad. En ese enfoque, la dinámica de cada población local sería independiente, y la de la metapoblación (el conjunto de poblaciones locales que ocupa la región) sería simplemente la suma de las dinámicas presentadas por las subpoblaciones locales. Si el movimiento de individuos hacia y desde las poblaciones locales ejerce una influencia significativa en la dinámica de estas, es necesario entonces formular un marco más amplio (Smith 2007).

En ese sentido, uno de los ecólogos más importantes en investigación de biología metapoblacional, Ilkka Hanski, propuso cuatro condiciones necesarias para la aplicación del término metapoblación a un sistema de poblaciones locales. Las cuatro condiciones son (Hanski 1999):

1. El hábitat adecuado se produce en diferentes parcelas que pueden estar ocupadas por poblaciones que se reproducen a nivel local.

2. Incluso las poblaciones más grandes tienen un riesgo considerable de extinción.

3. Las parcelas de hábitat no deben estar aisladas de tal modo que no permitan la recolonización tras una extinción local.

4. Las dinámicas de las poblaciones locales no están sincronizadas.

HETEROGENEIDAD ESPACIAL DE POBLACIONES

Además de las condiciones que definen una población, existe otro factor que influye de manera importante en la dinámica de una metapoblación: la heterogeneidad espacial de las poblaciones.

El entorno ecológico es discontinuo y varía en el tiempo de forma compleja e impredecible, por lo que es heterogéneo y en estado de no equilibrio (Sparrow 1999). Todos los sistemas ecológicos presentan niveles de heterogeneidad y fragmentación en diversas escalas, debido a procesos naturales o a actividades humanas, los que resultan fundamentales para la dinámica de poblaciones, ya que éstas suelen estructurarse espacialmente y responder según el ambiente en el que viven.

Andrewartha y Birch han simplificado esta estructura espacial mediante la definición de dos escalas: la escala local y la escala regional. La dinámica de una metapoblación, por tanto, se regula mediante dos grupos de procesos que operan a dos escalas espaciales diferentes (Andrewartha \& Birch 1984).

Por otra parte, Smith establece que, en la escala local o interterritorial, los individuos se mueven e interactúan entre sí en el transcurso de actividades como la alimentación y reproducción. El crecimiento y la regulación poblacional a nivel local se determinan según los procesos demográficos de natalidad y mortalidad (Smith 2007).

En tanto, en la escala metapoblacional o regional, que engloba el conjunto de poblaciones locales que componen la metapoblación, la dinámica se regula mediante la interacción de las poblaciones locales, es decir, a través de procesos de dispersión y colonización.

Debido a que todas las poblaciones locales enfrentan la probabilidad de extinguirse, la persistencia a largo plazo de la metapoblación depende del proceso de (re)colonización (Smith 2007). Si la tasa de recolonización excede a la tasa de extinción, la metapoblación puede persistir, aunque ninguna subpoblación puede sobrevivir en un fragmento particular a lo largo del tiempo (Mccullough 1996).

La colonización implica el movimiento de individuos desde las poblaciones locales existentes hacia fragmentos 
desocupados, a fin de formar nuevas poblaciones locales. Esta dispersión de individuos entre poblaciones locales es una característica clave de la dinámica metapoblacional, ya que, al no existir dispersión entre fragmentos, las poblaciones locales se desarrollarán de manera independiente.

$\mathrm{Al}$ respecto Smith señala, que en niveles de dispersión intermedios surge una dinámica en la que los procesos de extinción local y recolonización logran cierto equilibrio, en donde la metapoblación existe como una serie de fragmentos de hábitat ocupados y desocupados. Esto significa que el concepto de metapoblación se vincula a procesos de renovación poblacional (extinción y establecimiento de nuevas poblaciones) y el estudio de la dinámica metapoblacional es esencialmente el estudio de condiciones en las cuales estos dos procesos se equilibran (Smith 2007).

\section{CONSERVACIÓN EN METAPOBLACIONES}

ENFOQUE METAPOBLACIONAL EN ESPECIES CON PROBLEMAS DE CONSERVACIÓN

La Biología de la conservación tiene como objetivo la preservación de la diversidad biológica y ha surgido como respuesta a la degradación ambiental y extinción de especies. La acelerada alteración del hábitat y la introducción de especies invasoras exóticas han hecho que sea crucial el desarrollar modelos que predigan la probabilidad de extinción de especies raras o en peligro de extinción (Boyle 2003).

En este contexto, la teoría de metapoblaciones ha sido usada con frecuencia, en especies que se distribuyen en parches o aquellas especies que sufren constantemente alteraciones en su hábitat. Un claro ejemplo de aquello, lo constituye el estudio realizado en una metapoblación de venado bura (Odocoileus hemionus) en la Reserva de la Biósfera Mapimí, en México. Tras varios estudios en México, se estableció una evidente reducción en el área de distribución. Asimismo, un estudio sobre su abundancia, sugirió que sus poblaciones eran de densidades bajas y se encontraban fragmentadas. Lo anterior, motivó el estudio del venado bura en la Reserva de la Biósfera Mapimí, con el objetivo de determinar si se estaba en presencia de una metapoblación. Finalmente se pudo establecer que las poblaciones de venado bura en estudio si constituía una metapoblación (Sánchez \& Gallina 2000), lo cual resultó fundamental para generar un plan de conservación de esta especie.

En una situación similar, se encuentra actualmente el huemul del sur (Hippocamelus bisulcus). Esta especie se encuentra protegida en Chile por la Ley N. 19.473, prohibiéndose su caza, tenencia, posesión, captura, transporte y comercialización. Asimismo, a nivel internacional, se encuentra clasificada en la categoría de "en peligro de extinción" por la Lista Roja de Especies
Amenazadas (UICN). Adicionalmente, en junio de 2006, esta especie fue declarada Monumento Natural por el Ministerio de Agricultura de Chile y declarada en peligro de Extinción por el Ministerio Secretaría General de la Presidencia (CONAF 2010).

La distribución del huemul en territorio chileno era, originalmente, desde el río Cachapoal, en la Región de O'Higgins, hasta el estrecho de Magallanes. Sin embargo, en la actualidad se encuentra en las regiones del Bío Bío, los Ríos, Los Lagos, Aysén y Magallanes. Su distribución actual se caracteriza por poblaciones fragmentadas (Vila et al 2006), generadas por actividades humanas pasadas y presentes.

La reducción progresiva del número de individuos y de su distribución incrementó la fragmentación de la metapoblación de huemul de Chile y Argentina (López et al 2013).

Pérdida de hábitat, deterioro del hábitat invernal y la pérdida de conductas migratorias tradicionales son algunos de los factores negativos importantes que afectan a la mayoría de las subpoblaciones de huemules, especialmente en Chile central (Povilitis 1998).

\section{CONSERVACIÓN EX SITU}

La conservación de especies puede desarrollarse tanto dentro del hábitat natural o conservación in situ como fuera de este, denominado conservación ex situ. La conservación ex situ, en cautiverio o en colecciones, es la aplicación de una amplia variedad de recursos, técnicas e infraestructuras especializadas que contribuyen a la protección de la biodiversidad, recuperación y sobrevivencia de individuos o poblaciones fuera de su hábitat (Conabio 2009), con el fin de evitar la fragmentación y pérdida de hábitat provocadas por la acción humana (Wilson 2002).

El objetivo principal de la conservación ex situ es disminuir las posibilidades de extinción de especies con problemas de conservación, restableciendo poblaciones nuevas en el hábitat natural de estas especies. En particular, la conservación ex situ mantiene viables aquellas poblaciones de especies amenazadas (sobre todo cuando la amenaza es tan severa que no permite su subsistencia en condiciones in situ), provee servicios educativos y de concientización $\mathrm{y}$, promueve la investigación sobre especies y recursos genéticos (De Biodiversidad, E. R. 2001).

La conservación ex situ incluye colecciones de plantas y fauna, jardines botánicos, parques, centros de rehabilitación de animales nativos y colecciones de germoplasma de especies (Bonacic 2011). La reintroducción de individuos a su medio silvestre es una herramienta de conservación que aporta a la restauración ecológica, en tanto exista un hábitat disponible y las presiones que permitieron la disminución de individuos de la especie, ya no estén presentes.

Si bien el mecanismo de conservación in situ es el más utilizado, también se ha reconocido a los programas ex 
situ como un complemento esencial, sobre todo frente al evidente incremento en las tasas de extinción producto del cambio climático (Pritchard 2011).

Son variados los esfuerzos de conservación ex situ desarrolladas en el país y en el mundo para la protección y recuperación de especies en peligro. Las iniciativas van desde la crianza de anfibios y aves tanto en Chile como en el extranjero (Correa et al. 2016), hasta programas de conservación para grupos con difícil cuidado ex situ como cóndores y pudúes (Capdeville 2011; Reyes et al. 2011). En especial destaca el programa de manejo ex situ del anfibio Rhinoderma darwinni (rana de Darwin) gracias a un trabajo conjunto con zoológicos internacionales (Fabry-Otte \& Tirado-Sepúlveda 2012).

El presente trabajo busca aportar, desde el modelamiento matemático de sistemas biológicos, a la planificación de programas de conservación ex situ. Para ello se trabajará en base a una metapoblación que está extinguiéndose (como los ejemplos descritos del venado bura y huemul), y sobre la cual se aplicará una medida de conservación ex situ basada en un enfoque metapoblacional. Se pretende incorporar al modelo clásico de Levins (Levins 1969) una medida de conservación ex situ, consistente en la recolonización de cierto número de parches a intervalos constantes de tiempo, para posteriormente analizar la dinámica obtenida a través de simulaciones.

\section{EL MODELO}

Consideremos una metapoblación que, como tal, se encuentra distribuida en fragmentos o parches. Supongamos que el nivel de colonización y de extinción de cada parche es un proceso lento comparado con la dinámica que la abundancia poblacional desarrolla dentro de cada parche. Así, podemos pensar que, si un parche está ocupado, entonces el nivel de ocupación será cercano a la capacidad de carga que como sistema independiente posee el parche.

Para efectos del modelo a presentar, supondremos también que las tasas de colonización y de extinción son parámetros fijos e iguales para cada parche, independiente de las características específicas que cada uno de estos pueda tener.

Bajo estos supuestos, es posible considerar el modelo planteado por Richard Levins en su trabajo "Some demographic and genetic consequences of environmental heterogeneity for biological control" (Levins 1969):

$$
\left\{\frac{d u}{d t}=m u\left(1-\frac{u}{v}\right)-g u\right.
$$

Donde $u$ corresponde a la cantidad de parches ocupados, $v$ al total de parches que es posible ocupar y $m$ y $g$ son las tasas (fijas) de colonización y extinción respectivamente.

Supongamos que el modelo (1) describe la dinámica natural de una metapoblación que está extinguiéndose, es decir, la tasa de colonización es menor que la tasa de extinción $(m<g)$.

Nuestro interés es dotar al modelo (1) de una medida de conservación ex situ que permita que la población no se extinga y alcance, mientras se mantenga la medida, un nivel de equilibrio estable. Para esto, asumiremos que actúan dos escalas de tiempo, una continua para la dinámica metapoblacional natural, dada por (1), y la cual es comparativamente más lenta respecto a una segunda, la que describe la medida de conservación. Más precisamente, consideraremos que las intervenciones asociadas a la medida de conservación ex situ ocurren en una secuencia creciente de instantes $\left\{t_{k}\right\}$ que están igualmente espaciados en el tiempo, es decir, $t_{k+1}-t_{k}=\tau$, para cierto $\tau>0$ y para cada $k \geq 0$.

Para modelar la situación descrita, se considerarán herramientas propias de la teoría de ecuaciones diferenciales impulsivas.

El Modelo de Levins, presentado en (1), corresponde a una Ecuación Diferencial Ordinaria, cuya solución para la condición inicial $u(0)=u_{0}$, viene dada por:

$$
u(t)=\frac{(m-g) u_{0}}{\frac{m}{v} u_{0}+\left(m-g-\frac{m}{v} u_{0}\right) e^{-(m-g) t}}
$$

Ahora bien, como ya se explicó, con el propósito de evitar que la población total se extinga, consideraremos como medida de conservación la colonización ex situ de cierta cantidad de parches desocupados, a unidades constantes de tiempo, es decir, cada cierta cantidad de tiempo (fija y determinada con anterioridad) se colonizarán artificialmente parches vacíos con la cantidad necesaria de individuos para que este parche pueda considerase como ocupado.

Claramente, la cantidad de parches a colonizar no puede superar la cantidad de parches desocupadas. Más aún, por limitantes propias de la estrategia de conservación, no se cuenta con la posibilidad real de colonizar cualquier cantidad arbitraria de parches disponibles. Luego, consideraremos entonces que la cantidad de parches a colonizar en cada uno de los instantes en los que se lleve a cabo la estrategia de conservación dependerá de la cantidad de parches desocupados disponibles.

Por otra parte, es necesario que la metapoblación cuente con una cantidad inicial mínima de parches ocupados para que sea viable, y tenga sentido, el aplicar la medida de conservación y, en algún sentido, invertir los recursos asociados a esta. 
Estos hechos serán incorporados al modelo buscado, suponiendo que la cantidad de parches a colonizar mediante la medida de conservación ex situ es una función, $K$, de los parches desocupados, la cual definiremos por:

$$
K(d)=\left\{\begin{array}{ccc}
\alpha v \frac{d}{d_{0}+d} & \text { si } & d \leq d_{0} \\
0 & \text { si } & d>d_{0}
\end{array}\right.
$$

donde $d$ representa la cantidad de parches desocupados en el instante $t_{k}$ en el que se aplicará la medida de conservación, $\alpha v / 2$ es la cantidad máxima de parches que es posible colonizar artificialmente cada vez que se aplique la medida de conservación ex situ, con $0 \leq \alpha \leq$ $d_{0} / v$. Por otra parte, $d_{0}$ representa la cantidad máxima de parches desocupados que debe haber para que sea aplicable la medida de conservación, o equivalentemente, $u_{0}=v$ - $d_{0}$ es la cantidad mínima de parches ocupados que debe tener la metapoblación para que la medida de conservación se aplique.

Notemos que si bien la respuesta funcional $K$ definida es arbitraria, esta obedece a la idea de que la cantidad de parches a colonizar de manera artificial no tiene por qué ser necesariamente proporcional con respecto a la cantidad de parches desocupados. Por el contrario, entre más parches deban colonizarse artificialmente, es muy probable que más complejo sea el proceso, de ahí la forma de la función escogida. Resulta sencillo probar que para $0 \leq \alpha \leq d_{0} /$ $v$, se tiene que $K(d) \leq d$, para todo $d \in\left[0, d_{0}\right]$, lo que es coherente con la situación que se busca modelar (la cantidad de parches a ocupar no puede ser mayor a la cantidad de parches desocupados).

Queda abierta la inquietud sobre qué respuesta funcional es mejor, y que tanto varía la dinámica con respuestas funcionales de otro tipo.

Matemáticamente, la incorporación de esta medida de conservación puede ser expresada mediante un sistema de ecuaciones diferenciales impulsivas, suponiendo una secuencia de instantes $\left\{t_{k}\right\}$, igualmente espaciados en el tiempo $\left(t_{k+1}-t_{k}=\tau\right.$, para todo $\left.k\right) \mathrm{y}$ en los cuales se aplica la medida de conservación ex situ, colonizando de manera artificial $K(d)$ parches, con $d$ la cantidad de parches desocupados en ese instante.

Por lo tanto, la metapoblación se rige por una dinámica natural, determinada por la ecuación (1), pero, en los instantes de intervención $t_{k}$, se tendrá que $u\left(t_{k}^{+}\right)=u\left(t_{k}\right)+K(d)$, a causa de la medida de conservación ex situ aplicada. Es decir, después de cada instante de intervención, la cantidad de parches ocupados será igual a la cantidad de parches ocupados justo antes de la intervención, más los que se colonizaron por la medida de conservación ex situ.
De esta manera, considerando $d=v-u$, se obtiene el modelo:

$$
\left\{\begin{array}{c}
\frac{d u}{d t}=m u\left(1-\frac{u}{v}\right)-g u, \quad \text { si } t \neq t_{k} \\
u\left(t_{k}^{+}\right)=u\left(t_{k}\right)+K(v-u), \quad \text { si } t=t_{k}
\end{array}\right.
$$

Para efectos de este trabajo, nos interesa escribir $u\left(t_{k+1}\right)$ en función de $u\left(t_{k}\right)$, es decir, $u\left(t_{k+1}\right)=F\left(u\left(t_{k}\right)\right)$, con el fin de generar una sucesión de valores para la cantidad de parches ocupados, a partir de la cual sea posible obtener conclusiones respecto de la conservación de la metapoblación total.

Ya sabemos, por (2), que

$$
u(t)=\frac{(m-g) u_{0}}{\frac{m}{v} u_{0}+\left(m-g-\frac{m}{v} u_{0}\right) e^{-(m-g) t}}
$$

Luego, obtenemos que si $\mathrm{t}_{\mathrm{k}}<\mathrm{t}<\mathrm{t}_{\mathrm{k}+1}$, entonces:

$u(t)=\frac{(m-g) u\left(t_{k}^{+}\right)}{\frac{m}{v} u\left(t_{k}^{+}\right)+\left(m-g-\frac{m}{v} u\left(t_{k}^{+}\right)\right) e^{-(m-g)\left(t-t_{k}\right)}}$

donde, por (3) y (4), tenemos que

$u\left(t_{k}^{+}\right)=u\left(t_{k}\right)+\alpha v \frac{v-u\left(t_{k}\right)}{d_{0}+\left(v-u\left(t_{k}\right)\right)}$,

si $v-u\left(t_{k}\right) \leq d_{0}$, mientras que $\mathrm{u}\left(t_{k}^{+}\right)=u\left(t_{k}\right)$, si $v-u\left(t_{k}\right)>d_{0}$.

Así, si $v-u\left(t_{k}\right)>d_{0}$, entonces en realidad no hay intervención $\mathrm{y}$, por lo tanto, la dinámica metapoblacional será simplemente la determinada por el Modelo de Levins, (1), el cual converge a la extinción de la metapoblación. Pero, suponiendo $v-u\left(t_{k}\right) \leq d_{0}$, que es el caso que nos interesa, $\mathrm{y}$ evaluando en $\mathrm{t}_{\mathrm{k}+1}$, logramos generar la relación funcional:

$$
u\left(t_{k+1}\right)=\frac{(m-g) M}{\frac{m}{v} M+\left(m-g-\frac{m}{v} M\right) e^{-(m-g) \tau}}
$$

con $M=u\left(t_{k}\right)+\alpha v \frac{v-u\left(t_{k}\right)}{d_{0}+\left(v-u\left(t_{k}\right)\right)}$ y donde $m$ y $g$ corresponden a las tasas de colonización y extinción respectivamente, $v$ representa el total de parches disponibles, $\alpha v / 2$ es la cantidad máxima de parches que es posible colonizar en cada instante de intervención y $d_{0}$ es la cantidad máxima de parches desocupados aceptable para que se aplique la medida de conservación. Además, $\tau$ es la cantidad de unidades de tiempo que transcurren entre una intervención y la siguiente. 


\section{SIMULACIONES Y RESULTADOS PRINCIPALES}

En orden a obtener resultados y conclusiones a partir del modelo (5), pero evitando abusar de complejidades matemáticas fuera de los objetivos de este trabajo, es que se presentan a continuación una serie de simulaciones respecto de los posibles escenarios que la dinámica definida por (5) puede establecer para diversos valores en el espacio de parámetros asociado.

\section{EL MODELO DE LEVINS}

Una metapoblación extinguiéndose, en el sentido otorgado por el Modelo de Levins, (1), corresponde a una metapoblación en la que la cantidad de parches ocupados está convergiendo hacia el valor cero, independientemente de la cantidad de individuos que se encuentre distribuida en cada parche ocupado. Dentro del espacio de parámetros asociado al modelo, esto significa que la tasa de colonización $(m)$ es estrictamente menor que la tasa de extinción $(g)$. Un ejemplo gráfico de aquello se presenta en la Figura 1, donde se han considerado los valores $u_{0}=15, v=20, m=0,1$ y $g=0,15$.

Estos mismos valores serán considerados para todos los gráficos siguientes. De esta manera, podemos pensar que en todas las próximas simulaciones la metapoblación está extinguiéndose al ritmo indicado en la Figura 1. Las diferencias en el comportamiento de la dinámica, obedecerán entonces a los valores que se asignen a los parámetros asociados a la medida de conservación ex situ a aplicar.

\section{LA FUNCIÓN $K$}

Una medida de conservación ex situ, tal como se ha explicado en párrafos anteriores, corresponde a la incorporación de cierta cantidad de individuos a parches desocupados, de manera que estos puedan considerarse ocupados. De acuerdo a como se planteó el modelo a estudiar, la cantidad de parches a colonizar en cada intervención asociada a la medida de conservación ex situ viene dada por la función $K$ definida en (3).

Esta función $K$ dependerá de los parámetros $\alpha$ y $d_{0}$, donde $\alpha$ es un valor asociado a la cantidad máxima de parches que es posible colonizar artificialmente en cada instante de intervención, mientras que $d_{0}$ corresponde al valor máximo de parches desocupados que puede llegar a tener la metapoblación para que la medida de conservación sea aplicada. La existencia de estos parámetros se justifica en el hecho de que no es posible colonizar artificialmente más parches de los desocupados y que la aplicación de una medida de conservación parece no conveniente si ya existen demasiados parches desocupados. De estos dos hechos se obtiene que $0 \leq \alpha \leq d_{0} / v$.

Sin embargo, para las simulaciones siguientes supondremos $\alpha=d_{0} / v$, , bajo la idea de que la medida de conservación ex situ a aplicar pretende colonizar artificialmente la máxima cantidad de parches posibles, de modo de intentar asegurar que la medida de conservación sea lo más exitosa posible.

Algunas gráficas para la función $K$, considerando distintos valores para el parámetro $d_{0}$ se pueden observar en la Figura 2.

Es posible observar, en la Figura 2, que efectivamente $K(d)<d$, para todo $d$, tal como se explicó cuando se definió la función. Además, podemos notar que el proceso de colonización es menos efectivo a medida que mayor es la cantidad de parches a colonizar, supuesto que se consideró para definir la función $K$.

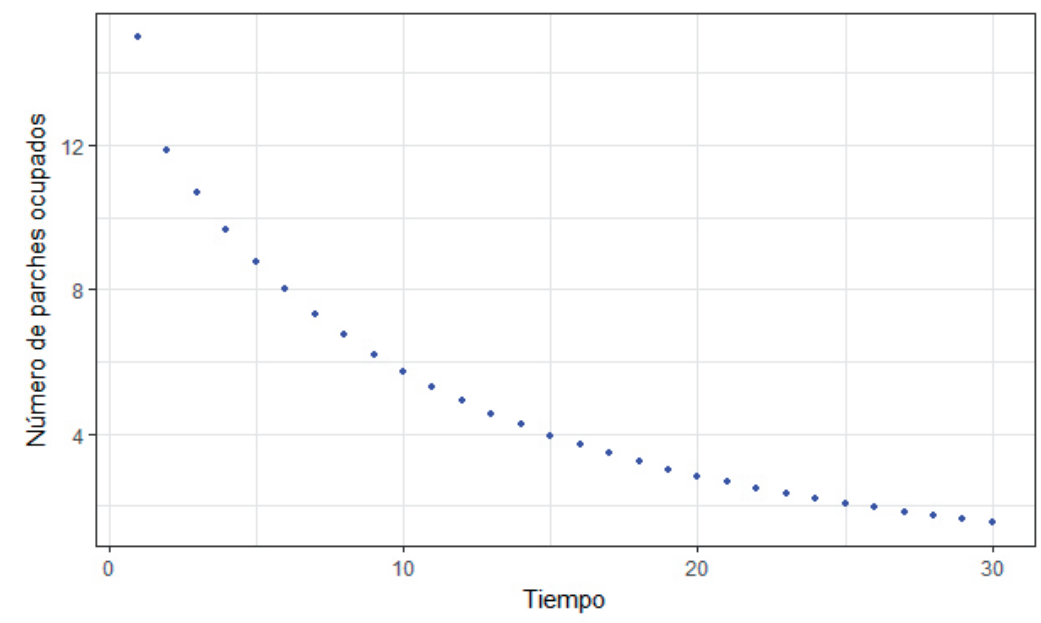

Figura 1. Dinámica de una metapoblación en proceso de extinción $(m=0,1$ y $g=0,15)$, de acuerdo al Modelo de Levins. / Dynamics of a metapopulation in the process of extinction ( $m=0.1$ y $g=0.15)$, according to the Levins Model. 


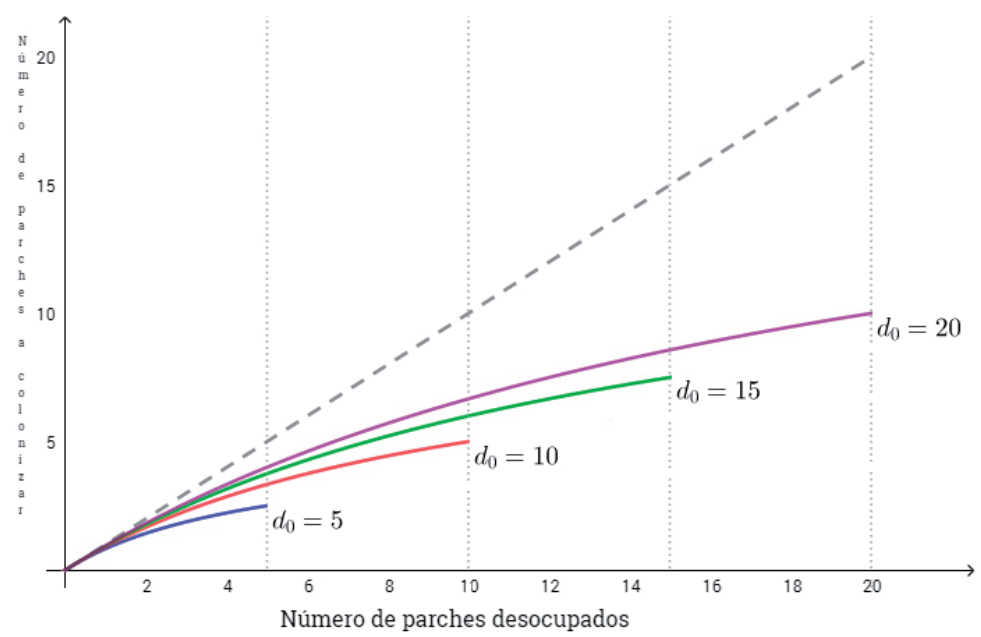

Figura 2. Gráficos asociados a la función $K$, para distintos valores de $d_{0}$. / Graphs associated with the $K$ function, for different values of $d_{0}$

EL MODELO DE LEVINS CON UNA MEDIDA DE CONSERVACIÓN EX SITU El modelo representado por la ecuación (4) entrega información respecto de una metapoblación cuya dinámica, en cuanto a la ocupación de los parches, esta modelada por el Modelo de Levins, (1), pero a la cual se le está aplicando una medida de conservación ex situ en términos de la función definida en (3), dando origen al modelo (5) que buscamos estudiar.

Es importante recordar que, para todas las simulaciones a presentar en los siguientes apartados, se tiene que $u_{0}=15$, $v=20, m=0,1, g=0,15$ y $\alpha=d_{0} / v$. Además, todas estas simulaciones muestran la dinámica de la metapoblación en función de los instantes $\left\{\mathrm{t}_{\mathrm{k}}\right\}$ (instantes de intervención), pues el modelo dado por (5) corresponde a un sistema discreto que entrega información respecto de la cantidad de parches ocupados en el instante previo a que se incorporen los individuos que poblaran los parches a colonizar artificialmente. Las simulaciones siguientes no entregan información respecto de la dinámica natural que determina el sistema entre dos momentos consecutivos de intervención, la cual sabemos está dada por (1) y su forma gráfica puede observarse en la Figura 1.

La Figura 3 muestra la dinámica determinada por el modelo (5), para distintos valores del espacio de parámetros.

En la Figura 3 se observa que a pesar de que, en todos los casos, la curva mantiene su comportamiento y decae, existen situaciones en las que esta converge hacia un valor distinto de 0 , señal clara de que se detuvo el inminente proceso de extinción, a diferencia de lo que ocurre en la situación original (Fig. 1).

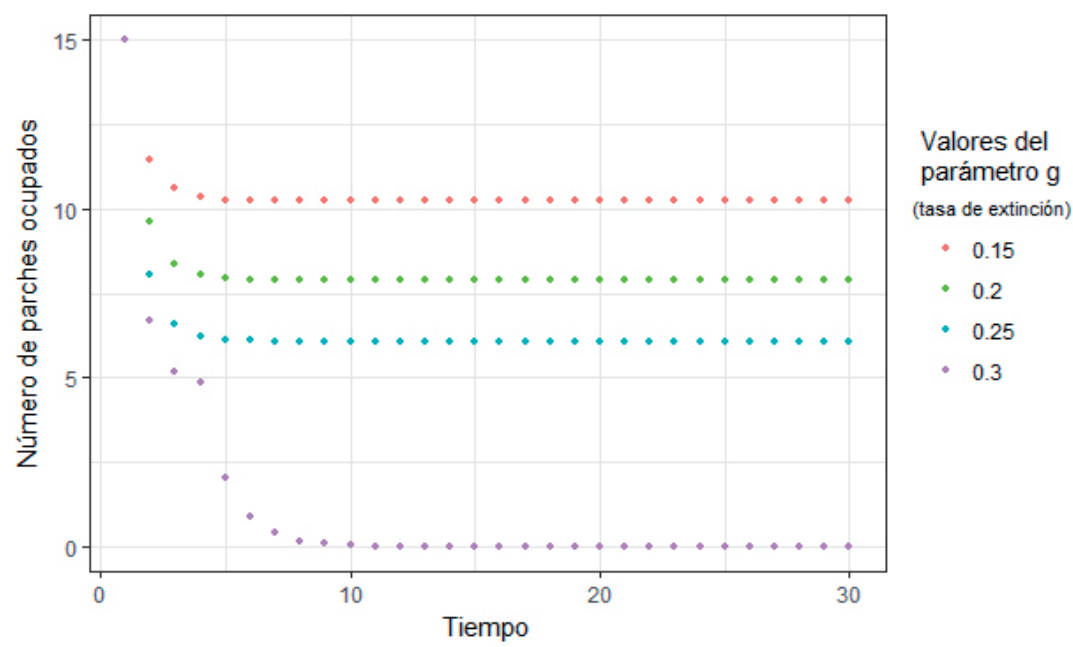

Figura 3. Modelo de Levins con una medida de conservación ex situ fija $\left(d_{0}=15, \tau=4\right)$, para distintos valores de la tasa de extinción $(g)$. $/$ Levins model with a fixed tex situ conservation measure $\left(d_{0}=15, \tau=4\right)$, for different values of the extinction rate $(g)$. 
Lo anterior permite suponer que la medida de conservación utilizada puede ser efectiva y estabilizar, tras algún paso en el tiempo, el sistema en un punto de equilibrio distinto de 0 , evitando la extinción total. Tenemos así, que la dinámica está determinada por un único punto de equilibrio globalmente estable (atractor), cuyo valor dependerá de los valores en el espacio de parámetros.

Las secciones siguientes intentan ver la relación entre este punto de equilibrio estable y los parámetros del modelo (5) que están asociados a la medida de conservación ex situ aplicada.

\section{El PARÁMETRO $d_{0}$}

El parámetro $d_{0}$ está asociado primeramente al máximo número de parches desocupados que puede tener la metapoblación para que se considere la aplicación de la medida de conservación ex situ. Viene a ser una medida relacionada con los criterios sobre la aplicación de una medida de conservación para una metapoblación en riego de extinción. Muestra de esto último, es que $\mathrm{K}(d)=0$ si $d$ $>d_{0}$, indicando que no se colonizará artificialmente ningún parche, si la cantidad de parches desocupados es mayor a la cota establecida por $d_{0}$.

Por otra parte, $d_{0}$ está asociado con el parámetro $\alpha$, mediante la relación $0 \leq \alpha \leq d_{0} / v$, la cual asegura que $K$ $(d)<d$ para todo $d$, es decir, asegura que la cantidad de parches a colonizar artificialmente es menor a la cantidad de parches desocupados disponibles. Ahora bien, si suponemos que el propósito es colonizar la mayor cantidad de parches posibles, en búsqueda de la conservación de la metapoblación, tendríamos que $\alpha=d_{0} / v$, donde $v=20$ (parámetro ya definido anteriormente para las simulaciones presentadas). La Figura 4 muestra la cantidad de parches ocupados en la metapoblación para valores de $d_{0}$ entre 0 y 9 .

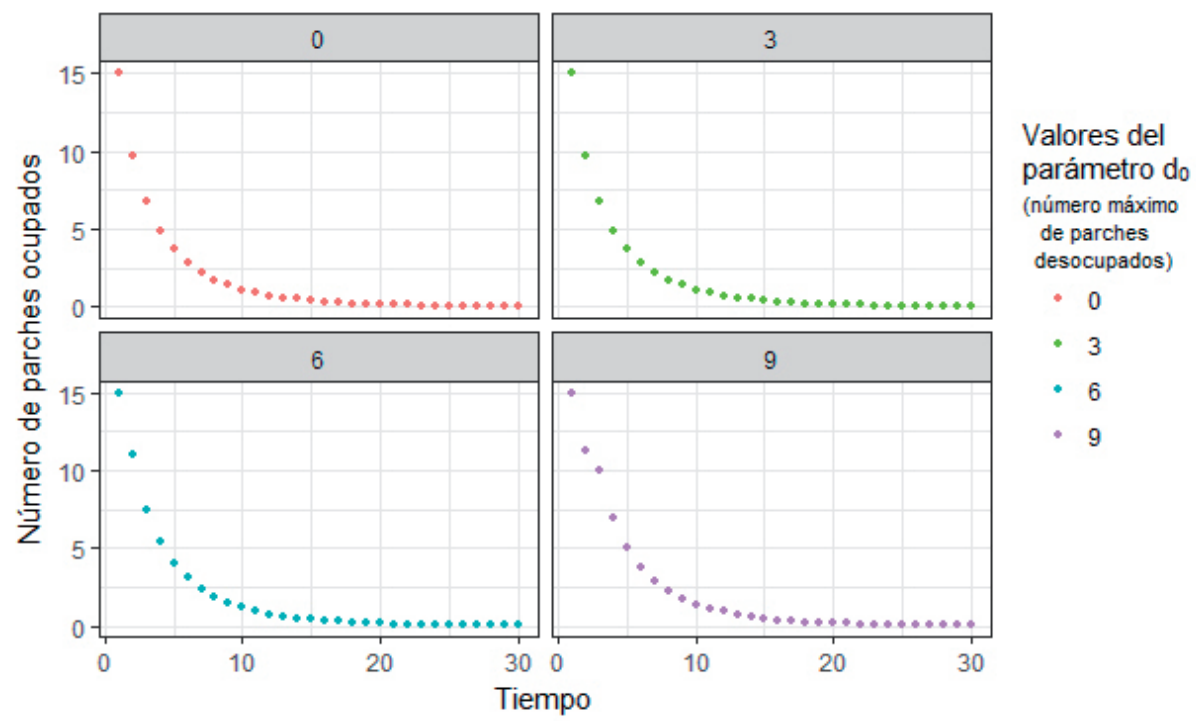

Figura 4. Modelo de Levins con una medida de conservación ex situ para valores de $d_{0}$ entre 0 y 9 . / Levins model with an ex situ conservation measure for values of $d_{0}$ between 0 and 9 .

Podemos notar que, en todos los escenarios, la cantidad de parches ocupados converge a 0 , indicando la extinción de la metapoblación, es decir, para el set de parámetros escogidos, un valor de $d_{0}<10$ no es suficiente para conseguir que la metapoblación no se extinga por completo. En otros términos, aplicar la medida sólo si la cantidad de parches desocupados es menor a 10 (la mitad del total disponible), con una cantidad máxima de parches a colonizar menor a $\alpha \nu / 2=5$ no es suficiente para lograr el éxito de la medida de conservación.

Sin embargo, la Figura 5 grafica la cantidad de parches ocupados en la metapoblación para valores de $d_{0}$ entre $11 \mathrm{y}$ 20 , con una dinámica muy distinta a la anterior.
Para estos valores en el espacio de parámetros, tenemos convergencia a un punto de equilibrio estable distinto de 0 (más aún, cercano a 10 ), que permite concluir que la medida de conservación es exitosa, evitando la extinción de la metapoblación.

Por otra parte, resulta interesante notar que el valor al cual converge la cantidad de parches ocupados para estos valores de $d_{0}$ no muestran mayores diferencias entre sí, por ende, parece que si bien existe una condición umbral para $d_{0}$ que define un cambio importante en la dinámica (para este caso cercana a $d_{0}=10$ ), no es necesariamente cierto que valores más altos para $d_{0}$ generen puntos de equilibrio notablemente mayores. Probablemente entonces, en el contexto del valor 
al cual converge la cantidad de parches ocupados, no existe mucha diferencia entre considerar $d_{0}$ como un valor cercano a 11, o como un valor cercano a 20 . Sin embargo, este hecho si podría marcar una considerable diferencia en cuanto a los recursos necesarios para la implementación de la medida de conservación ex situ seleccionada.

\section{EL PARÁMETRO $\tau$}

La Figura 6, presentada a continuación, muestra la cantidad de parches ocupados en los distintos instantes de intervención, para diferentes valores del parámetro $\tau$, el cual corresponde a la cantidad de unidades de tiempo existentes entre una intervención y la siguiente $\left(\tau=t_{\mathrm{k}+1}-\mathrm{t}_{\mathrm{k}}\right)$.

Podemos notar entonces, que el punto de equilibrio al cual tiende la cantidad de parches ocupados depende del tiempo transcurrido entre cada intervención. Más aún, la simulación da cuenta de valores de $\tau$ para los cuales la metapoblación se extingue, y pareciese, al menos por lo mostrado en la gráfica, que el valor al cual converge la sucesión de punto es más bien sensible al valor asignado para el parámetro $\tau$.
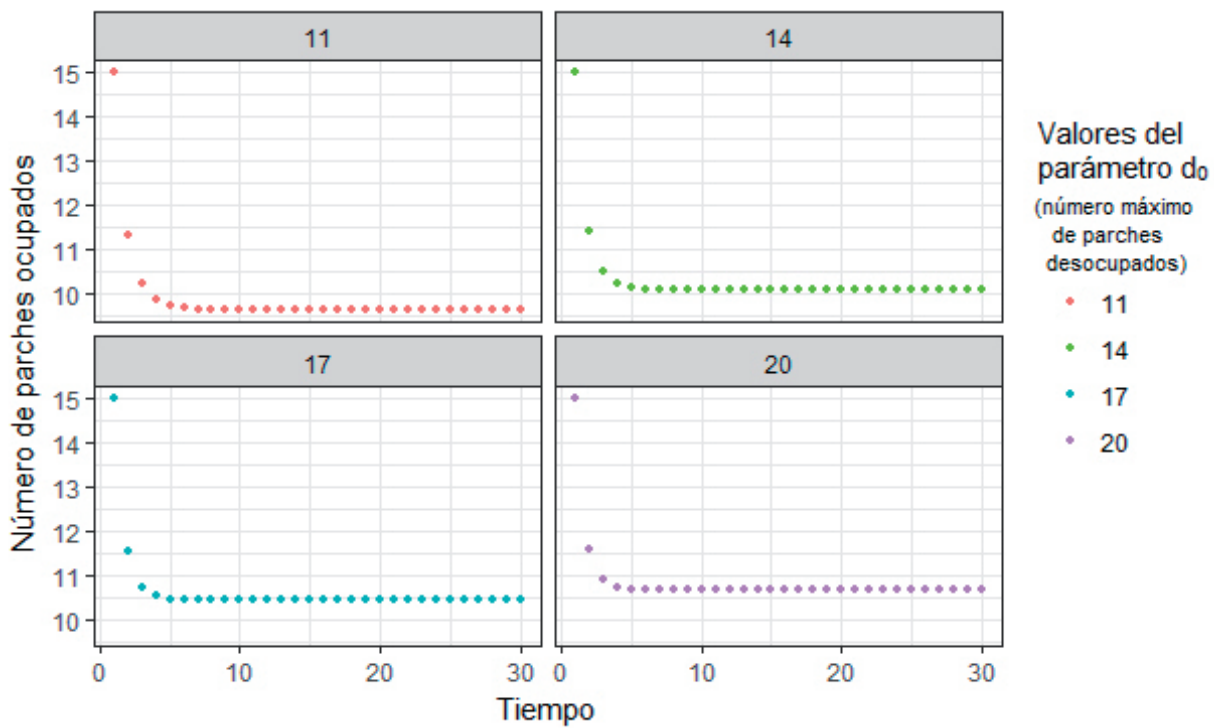

Figura 5. Modelo de Levins con una medida de conservación ex situ para valores de $d_{0}$ entre 11 y 20 . / Levins model with an ex situ conservation measure for values of $d_{0}$ between 11 and 20 .

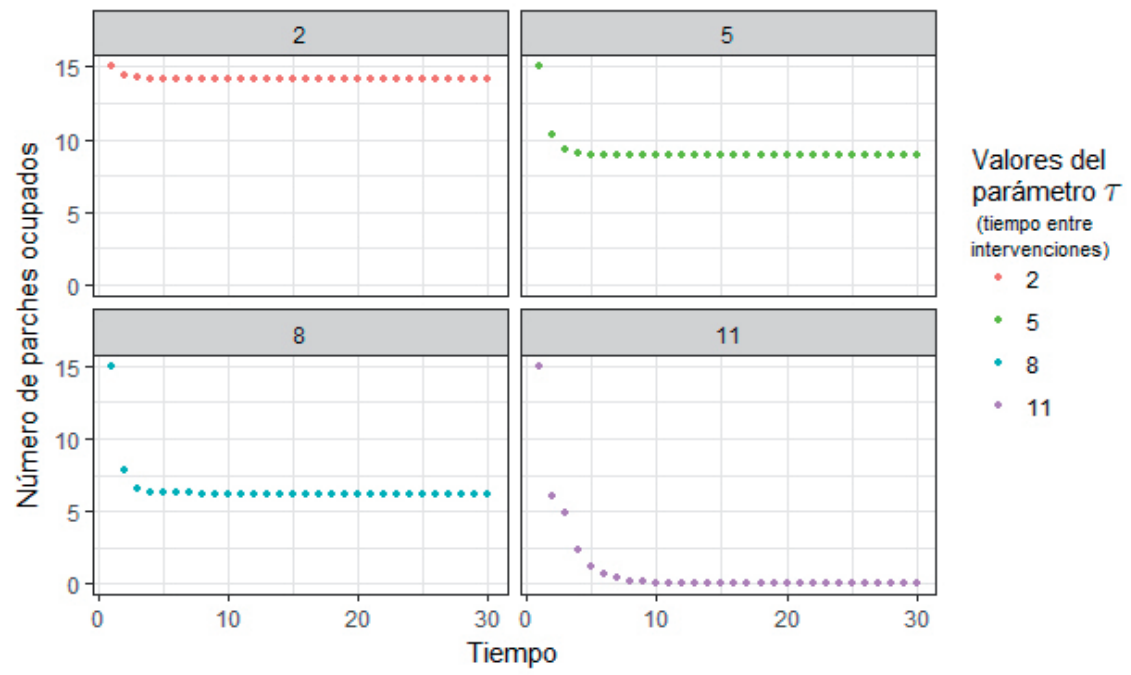

Figura 6. Modelo de Levins con una medida de conservación ex situ para distintos valores de $\tau$. / Levins model with an ex situ conservation measure for different values of $\tau$. 
Este último hecho puede entenderse bajo la idea de que un valor de $\tau$ mayor implica que la metapoblación tiene mayor tiempo para desarrollarse en términos de la dinámica asociada al Modelo de Levins y, por tanto, la cantidad de parches ocupados decrece. Consecuencia de esto es que, al momento de la intervención, la cantidad de parches a colonizar, además de verse afectada por las complejidades del proceso de colonización expresado en términos de la función $K$, no alcancen a oponerse al efecto del decaimiento natural que la metapoblación está desarrollando. O incluso, no contar con la cantidad de parches ocupados mínima para poder aplicarse la medida de conservación.

\section{DISCUSIÓN}

Un enfoque particular de conservación es el que se desarrolla cuando la población en estudio corresponde a una metapoblación (Hanski 1997). Si estamos frente a una metapoblación, entonces esta puede ser considerada como una colección de parches (ocupados o desocupados) y, por lo tanto, las medidas de conservación pueden enfocarse en la cantidad de parches ocupados, más que en la cantidad total de individuos presentes en la población o en cada parche en particular.

Una medida de conservación posible, conocida como conservación ex situ, consiste en colonizar artificialmente cierta cantidad de parches desocupados, de modo que estos pasen a considerarse como ocupados (Bonacic 2011; Conabio 2009). Donde la cantidad de individuos a incorporar en cada parche para que este sea considerado como ocupado dependerá de cada metapoblación.

Bajo esta idea y basados en el modelo clásico de Levins (Levins 1969), se diseñó, programó y analizó un modelo matemático que suponía una metapoblación extinguiéndose a la cual se aplica una medida de conservación ex situ, consistente en la colonización artificial, a instantes constantes de tiempo, de cierta cantidad de parches, determinados por una función particular.

La información entregada por las distintas simulaciones presentadas indica, primero que nada, que efectivamente una medida de conservación ex situ puede, eventualmente, ser exitosa en su afán de evitar que una metapoblación se extinga. Esto se refleja en que existen escenarios en el espacio de parámetros que define el modelo, para los cuales el sistema converge a un punto de equilibrio estable, y distinto de 0 , tras la aplicación de la medida de conservación.

Por supuesto que el valor del punto estable al cual converge el sistema dependerá de los valores asignados a los parámetros del modelo. Sin embargo, el valor $d_{0}$, que representa la cantidad máxima de parches desocupados que debe haber para que la medida de conservación se aplique, y que además se logró relacionar con la cantidad máxima de parches a colonizar en cada instante de intervención, posee una condición umbral que divide la dinámica entre la extinción (convergencia a 0 ) y la estabilidad en un punto de equilibrio distinto, el cual si bien varía respecto del valor exacto de $d_{0}$, la variación no es mayormente relevante (al menos numéricamente).

Esto último podría sugerir que existe un valor $d_{0}$ para el cual la metapoblación no se extingue tras la aplicación de la medida de conservación ex situ, pero que no genera resultados considerablemente más exitosos, en cuanto a la cantidad de parches ocupados, que otros valores mayores a él, siendo innecesario entonces la colonización de una cantidad de parches mayor a la calculada a partir del valor $d_{0}$ mencionado. De esta manera, es posible establecer un valor para $d_{0}$ que optimice el uso de los recursos asociados a la aplicación de la medida de conservación.

Por otra parte, el parámetro $\tau$, que mide las unidades de tiempo que transcurren entre una intervención y la siguiente, también influye en el comportamiento de la dinámica, pero en este caso, como era esperable, a mayor valor de $\tau$, menor será el valor al cual converge la cantidad de parches ocupados, existiendo un valor particular para $\tau$, a partir del cual la metapoblación termina extinguiéndose totalmente.

El determinar los valores óptimos que se mencionan en los párrafos anteriores, y hacer un estudio de estos, obedece a un trabajo matemático más detallado, el cual queda planteado como un posible camino a seguir.

Finalmente debemos señalar, aunque resulte claro, que, en el modelo planteado, para valores cualquiera en el espacio de parámetros, el dejar de aplicar la medida de conservación ocasionará inevitablemente que la metapoblación retome su dinámica natural, haciendo que la cantidad de parches ocupados converja a 0 y por ende, que la metapoblación se extinga. Por lo tanto, para que la medida de conservación aplicada deje de ser necesaria en algún momento, esta debe ir acompañada con estrategias que permitan que la tasa de decaimiento $(m-g)$ llegue a tomar un valor positivo. De acuerdo a nuestro entender, existen estrategias que apuntan a mejorar los parches que ocupan ciertas metapoblaciones, a fin de que estas se mantengan en el tiempo.

Queda entonces, como desafío último, el estudio de un modelo matemático como el presentado en este trabajo, pero en el que la tasa $m$ - $g$ corresponda a una función creciente que en algún instante de tiempo tome valores positivos. De esta manera, se podrían establecer conclusiones respecto al tiempo que debe aplicarse la medida de conservación $e x$ situ, la cantidad de parches a colonizar artificialmente (la cual incluso podría considerarse variable) e incluso como distribuir los recursos entre la medida de conservación ex situ y la estrategia de mejora de los parches. 


\section{REFERENCIAS}

Andrewartha, G., Birch, L. 1984. The ecological web. University of Chicago Press, Chicago. 520 pp.

Bonacic, C. 2011. La conservación ex situ como herramienta de conservación biológica. En: Iriarte, A., Tala, C., González, B., González, G., Maino, M. (Eds) Cría en cautividad de Fauna Chilena: 249-260. Servicio Agrícola y Ganadero, Duplika, Chile, Santiago.

BoyLe, O. 2003. La aplicación de la teoría de metapoblaciones para la conservación de plantas raras: el caso de Polygonella basiramia en el Matorral de Florida, Estados Unidos. Ecosistemas 12:1-5.

Capdeville, A. 2004. Proyecto de conservación Condor Andino de Argentina: Una metodología de cría ex situ para la conservación in situ, el nuevo rol que los zoológicos están llamados a cumplir en la conservación global. En: Iriarte, A., Tala, C., González, B., González, G., Maino, M. (Eds) Cría en cautividad de Fauna Chilena: 249-260. Servicio Agrícola y Ganadero, Duplika, Chile, Santiago.

CONABIO. 2009. Capital natural de México, vol. II : Estado de conservación y tendencias de cambio. Comisión Nacional para el Conocimiento y Uso de la Biodiversidad, México.

Corporación Nacional Forestal. 2010. Plan Nacional de Conservación del Huemul (Hippocamelus bisulcus, Molina 1782) en Chile. Unidad Comunicación y Prensa, SAG. Chile.

Correa, C., Donoso, J., Ortiz, J. 2016. Estado de conocimiento y conservación de los anfibios de Chile: una síntesis de los últimos 10 años de investigación. Gayana 80(1):103-124.

Fabry-Otte, M., Tirado-Sepúlveda, M. 2012. Rol del ZoológicoNacional en la conservación de los anfibios de Chile: el ejemplo de Rhinoderma darwinii. En: SotoAzat, C., Valenzuela-Sánchez, A. (Eds) Conservación de Anfibios de Chile: 84-86. Universidad Nacional Andrés Bello, Quad/graphics Chile S.A., Santiago

Hanski, I., Simberloff, D. 1997. The metapopulation approach, its history, conceptual domain, and application to conservation. En: Hanski, I., Gilpin, M. (Eds) Metapopulation biology: ecology, genetics, and evolution. Academic Press, San Diego, USA.
HANSKI, I. 1999. Metapopulation ecology. Oxford University Press, New York, USA. 328 pp.

Levins, R. 1969. Some Demographic and Genetic Consequences of Environmental Heterogeneity for Biological Control. Bulletin of the Entomological Society of America 15:237240.

López, R., Corti P., SAucedo, C. 2013. Estado y Distribución de las Poblaciones de Huemul Existentes en el Predio Fiscal El Azul y áreas Colindantes. Corporación Chile Ambiente.

McCullough, D. 1996. Metapopulations and Wildlife Conservation. Island Press, Washington.

Povilitis, A. 1998. Characteristics and conservation of a fragmented population of huemul Hippocamelus bisulcus in central Chile. Biological Conservation 86:97-104.

Pritchard, D., Fa, J., Oldfield, S., Harrop, S. 2011. Bring the captive closer to the wild: redefining the role of ex situ conservation Fauna \& Flora International. Oryx 46(1):1823.

Reyes, E., Lobos, A., Cortes, R., Bubenik, G., Schams, D., Bartos, L., Acuña, A. 2004. Programa Conservación de la especie nativa Pudu pudu, Molina (Mammalis, Cervidae). En: Iriarte, A.; Tala, C.; GonzÁlez, B.; GonzÁlez, G.; Maino, M. (Eds) Cría en cautividad de Fauna Chilena: 363-370. Servicio Agrícola y Ganadero, Duplika, Santiago, Chile.

Rodríguez, D. 2006. Modelos de Metapoblaciones y de la dinámica espacio-temporal de Comunidades. Publicaciones de la Universidad de Alicante, Alicante, España. 284 pp.

Sánchez, G., Gallina, S. 2000. La metapoblación del venado bura en la reserva de la biosfera Mapimí, México: Consideraciones para su conservación. Cuadernos de biodiversidad 22:7-15.

Sмiтh, T., Sмiтн, R. 2007. Ecología. Pearson Educación. Madrid, España. 776 pp.

SPARrow, A. 1999. A heterogeneity of heterogeneities. Trends in Ecology \& Evolution 14:422-423.

Vila, A., López, R., Pastore, H., Faúndez, R., Serret, A. 2006. Current distribution and conservation of the huemul (Hippocamelus bisulcus) in Argentina and Chile. Mastozoología Neotropical 13:263-269.

Wilson, E., Perlman. D. 2002. Conserving Earth's Biodiversity: An interactive learning experience for stuying conservation biology. Interactive CD-Rom. Island Press, Washington D.C.

Recibido: 30.12 .2017

Aceptado: 28.01.2019 